Jpn. J. Med. Sci. Biol., 49, 151 - 165, 1996.

\title{
HEMATOLOGICAL IMPAIRMENTS IN RECURRENT PLASMODIUM VIVAX INFECTED PATIENTS
}

\author{
Ramasamy SELVAM and Govindarajan BASKARAN
}

Department of Medical Biochemistry, Post Graduate Institute of Basic Medical Sciences, University of Madras, Taramani Campus, Madras 600113, India

(Received November 29, 1995. Accepted August 28, 1996)

SUMMARY: The hematological parameters were assayed in Plasmodium vivax patients with only one infection, two infections, three infections and more than three malarial infections during a period of six months. A steady fall in the levels of hemoglobin as well as packed cell volume (PCV) level was observed with increasing number of infections. The malarial patients showed a progressive decrease in RBC level with increasing number of attacks. The decrease in the hematological indices was statistically significant at all levels of parasitemia. There was a marked increase in the osmotic fragility of the malarial erythrocytes when compared to that of controls. During repeated malarial attacks, significant decrease in $\mathrm{MCH}(\mathrm{p}<0.05)$ and $\mathrm{MCHC}(\mathrm{p}<0.01)$ and increase in the MCV level $(p<0.05)$ and Heinz body formation $(p<0.001)$ were observed. Parasite density significantly influenced the fragility of the erythrocytes, Heinz body formation, MCV, MCH and MCHC levels. Thus, the erythrocytes of the patients repeatedly infected with Plasmodium vivax parasite are subjected to structural and functional impairment, ultimately culminating in anemia.

\section{INTRODUCTION}

Various in vivo and in vitro observations suggest that oxidative mechanisms play a role in the host defense against parasitic infections such as malaria (1). Red cell damage by oxidant stress is generally thought to be the net result of 
two processes: the oxidation of hemoglobin followed by denaturation of methemoglobin to hemichromes and free polyunsaturated fatty acid side chains of the membrane lipids; the reduced thiol groups and other susceptible amino acid chains of the membrane proteins.

The erythrocytes are at increased risk from oxidative processes for a variety of reasons. When it is continuously exposed to high oxygen tensions, hemoglobin is prone to autoxidation and can function as an oxidase and a peroxidase (2). The malaria parasite derives a number of biochemical advantages from its sojourn within the erythrocytes of the host. All these reports strongly emphasize that malarial infection is incriminated as a causative factor underlying erythrocyte metabolic impairment.

Our earlier studies have revealed augmented lipid peroxidation and poor antioxidant status in the palsma of Plasmodium vivax malaria patients (3). The current study focusses the influence of Plasmodium vivax recurrence on the hematological indices.

\section{MATERIALS AND METHODS}

Age-and-sex matched healthy controls who were residents of the endemic area with similar socio-economic status formed the control group $(n=63)$. Patients who had similar symptoms of malaria but were reported by negative for any parasites in the peripheral blood smear formed the negative control group $(n=20)$.

Only male patients $(n=172)$, with ages ranging from $14-40$ years, were included for this study, owing to a higher rate of malaria positivity, social convenience and easy follow-up. The patients,

1. had the trophozoite and gametocyte stages of $P$. vivax in the peripheral blood smear.

2. were not diabetic and had not undergone any treatment prior to sampling.

3. had a single or multiple attacks of malaria within a period of six months. Based on the patient's medical record, the subjects were grouped as follows:
a. Group I
- single infection
b. Group II
- two infections
c. Group III
- three infections
d. Group IV
- more than three infections. 
Blood was drawn by slow venepuncture and transferred into a tube containing ethylenediaminetetraacetate (EDTA, $10.5 \mathrm{mg} / 7.0 \mathrm{ml}$ ). The anticoagulated whole blood was used to assay for the hematological indices.

Hemoglobin was estimated by the method of Drabkin and Austin (4). Packed cell volume (5) and erythrocyte count (6) were also assayed. Erythrocyte indices (MCV, $\mathrm{MCH}$ and $\mathrm{MCHC}$ ) and leukocyte count were estimated by the methods of Wolf et al. (7) and Miale (8), respectively. Fragility of the erythrocytes in hypotonic saline was assessed by the method of Dacie (9). Heinz body formation was estimated in the erythrocytes (10). Samples of the malarial patients were further classified on the basis of parasite density estimated by the method of Seshadri et al (11). Quality control of blood parameters was checked periodically with a reference laboratory of Corporation of Madras Clinic.

Student Newman Keuls test was used to compute statistically for significant differences in the above population. Pearson's correlation coefficient ' $r$ ' was arrived at to assess the degree of linear association among the different variables taken two at a time.

\section{RESULTS}

The parasite density in the malarial blood samples was found to be variable and a maximal parasite density was observed to be $780 / \mathrm{mm}^{3}$ of blood. Hence, malaria patients were classified based on the parasite density namely $<250,250-$ $450,450-650$ and $>650$. From Table I, it is evident that the parasite density increased with repeated attacks. In the first and second attack patients, low parasitemia was mostly encountered.

Table II depicts the hematological indices namely blood hemoglobin $(\mathrm{Hb})$, packed cell volume (PCV), erythrocyte count (RBC), Heinz bodies and osmotic fragility of erythrocytes. A significant fall in the $\mathrm{RBC}$ count, $\mathrm{Hb}$ content as well as PCV was observed with increasing number of malarial infections.

There was a marked increase in the osmotic fragility of the malarial erythrocytes (group I - $p<0.05$; group II $-\mathrm{p}<0.01$; group III $-\mathrm{p}<0.01$ and group IV $-p<0.001$ ) when compared to that of controls. However, MCH and MCHC did not show any significant change upto two infections but in more than two repeated infections both decreased $(p<0.05)$. An increase in MCV $(p<0.05)$ was observed in all groups of patients. Repeatedly infected patients (Group III and IV) 


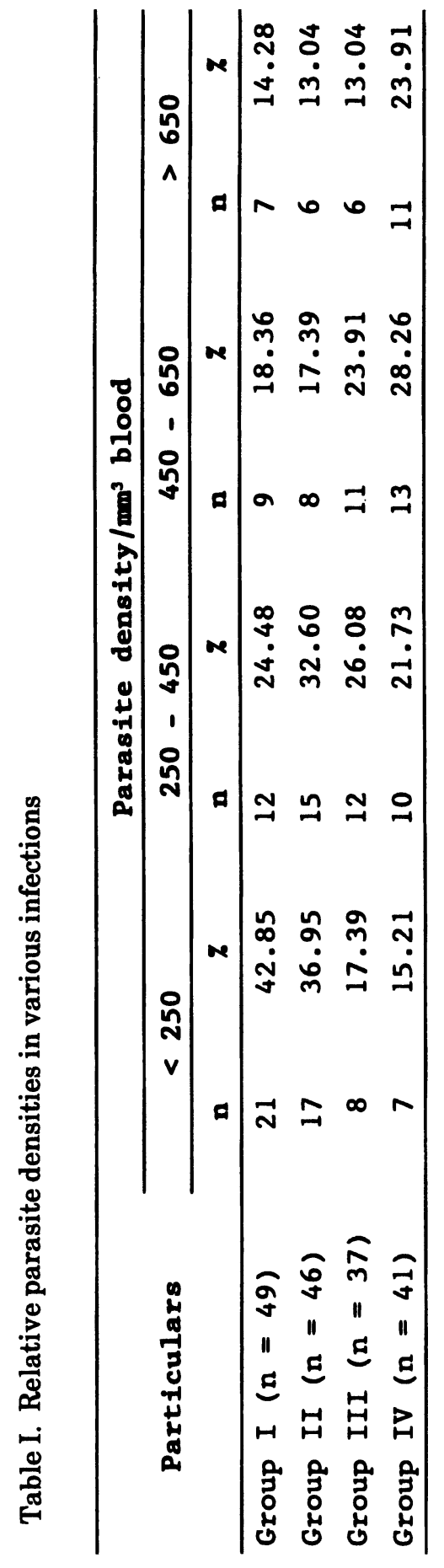




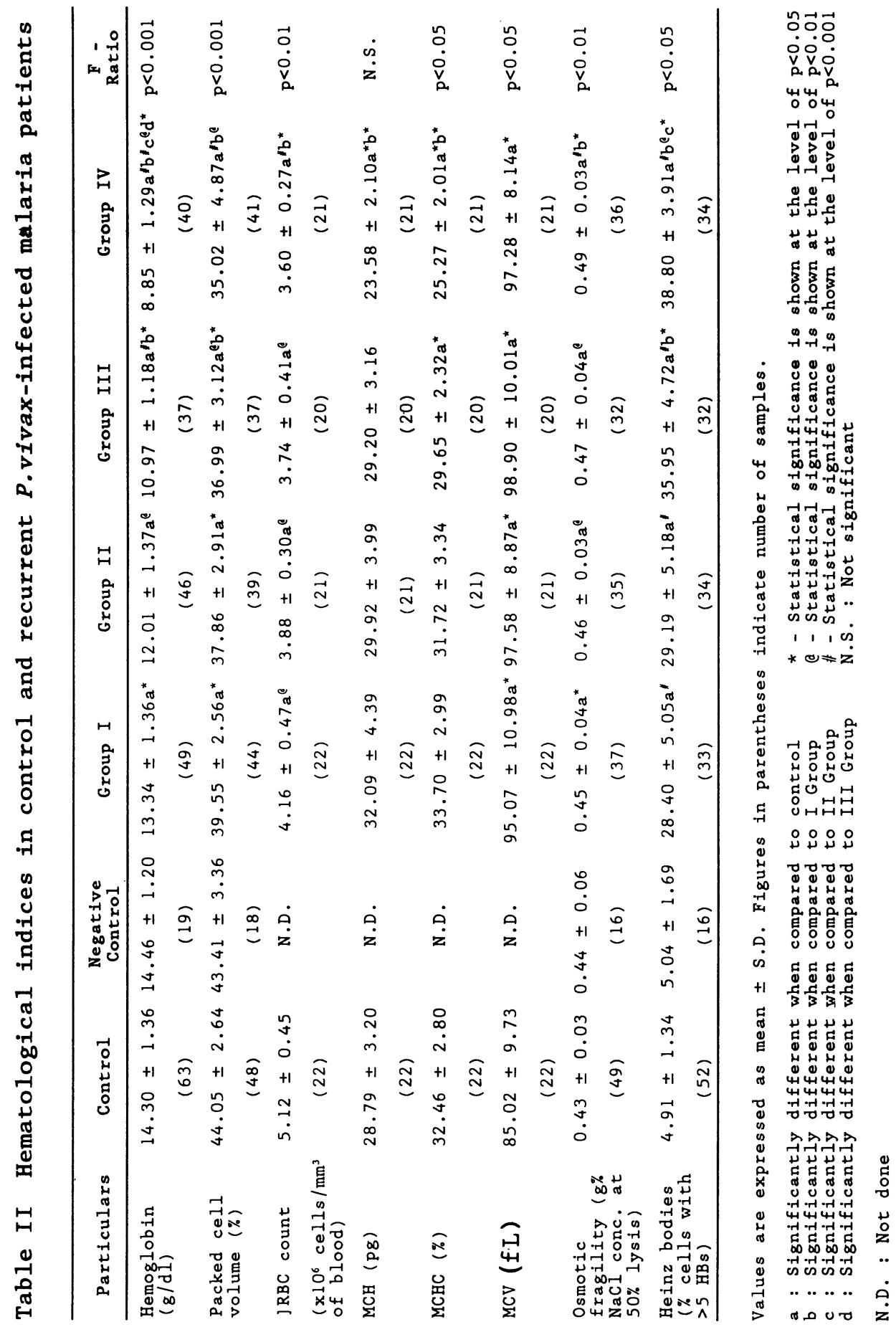



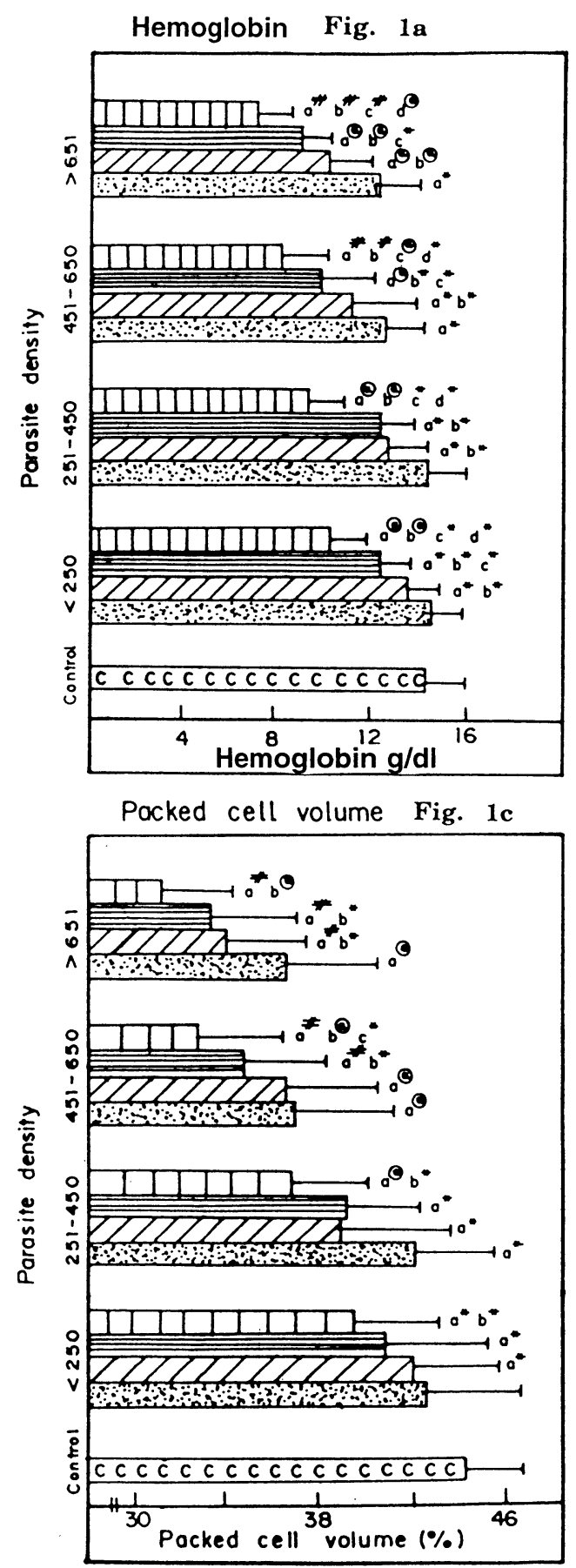

RBC Fig. 1b

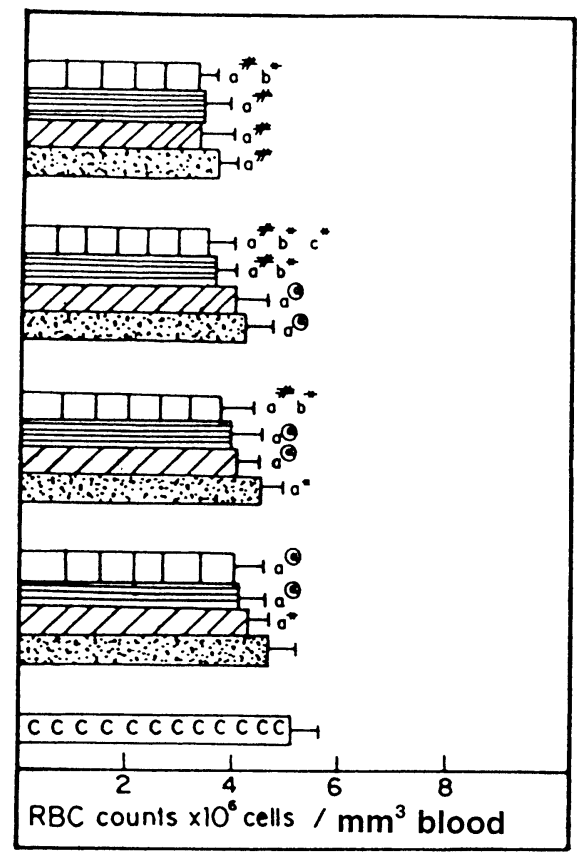

Osmotic fragility Fig. 1d

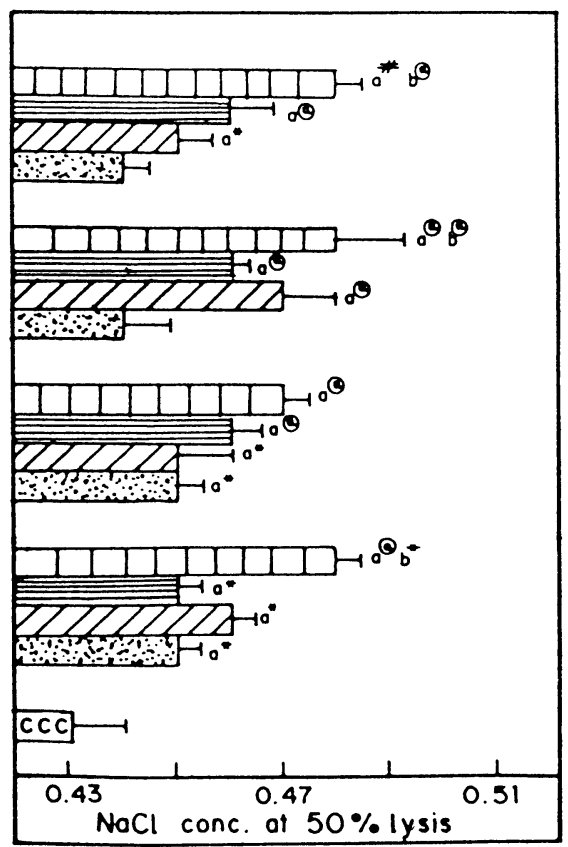


Fig.le

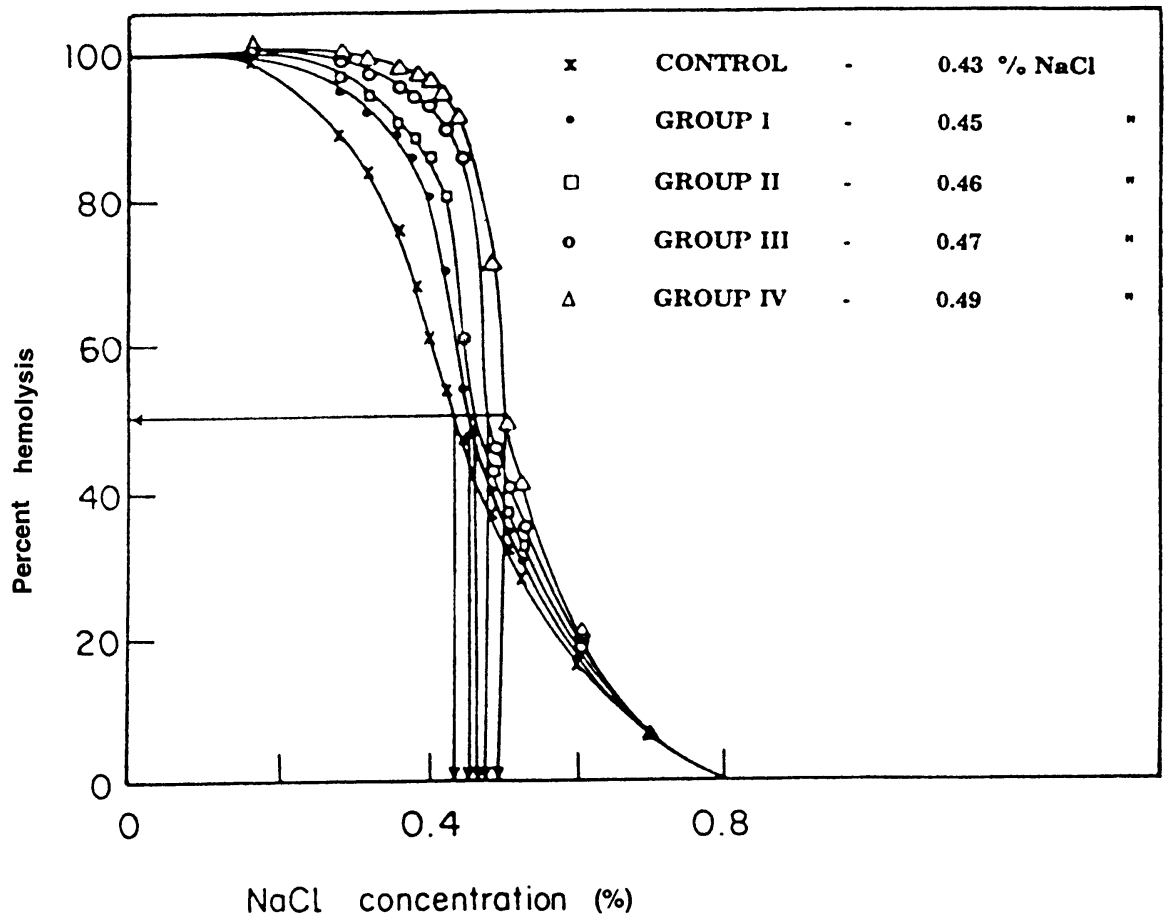

Fig. 1a, 1b, 1c, 1d. Effect of parasitemia on hemoglobin, RBC, packed cell volume and osmotic fragility.

Fig. 1e. Typical osmotic fragility curves of control, groups I, II, III and IV $P$. vivax patients.

manifested pronounced Heinz body formation $(p<0.001)$ when compared to single-infected patients.

When the effect of parasitemia on the hematological parameters was analyzed, there was a progressive decrease in $\mathrm{Hb}$ levels with increasing parasitemia (Fig. 1a). Parasitemia did not significantly influence the RBC count in the repeatedly infected malarial patients (Fig. 1b).

In patients with multiple attacks, PCV showed a significant decrease at all levels of parasitemia. However, fragility of the erythrocytes was increased signficantly at all levels of parasitemia during malarial infection when compared to that of control (Fig. 1d). With repeated infections, the erythrocytes became 
more fragile as evidenced by their lysis at different percentages of saline (group I 0.45; group II - 0.46; group III - 0.47; group IV - 0.49 and control - 0.43; Fig. 1e).

At a parasitemia of $<450 / \mathrm{mm}^{3}$, the patients with more than three infections showed a marked decrease $(\mathrm{p}<0.05)$ in $\mathrm{MCH}$ level when compared to the healthy individuals. In contrast, $\mathrm{MCHC}$ levels were elevated from the normal levels in the multiple-attack patients. Compared to the controls, the MCV levels were found to increase in all malarial patients irrespective of number of infections (Fig. 2c). When the parasitemia exceeded $450 / \mathrm{mm}^{3}$, Heinz body formation was augmented during all attacks. Patients with multiple attacks had significantly higher Heinz bodies even at low parasite counts than the patients with the first and second attacks (Fig. 2d).

Table III presents the correlation between $\mathrm{Hb}$ and other hematological indices. $\mathrm{Hb}$ levels did not correlate with any of the blood profiles in the control subjects. In the fresh malaria patients, it showed a direct correlation with RBC $(p<0.05)$, but an inverse relationship with Heinz bodies $(p<0.05)$. Hb correlated positively with PCV and RBC levels $(p<0.05)$ and negatively with erythrocyte osmotic fragility and Heinz bodies $(p<0.05)$ in the case of the IInd attack patients. During the IIIrd attack, $\mathrm{Hb}$ level of the malaria patients manifested a direct correlation with PCV and RBC contents at 5\% level, and inversely with parasite count $(p<0.05)$, MCV $(p<0.05)$, osmotic fragility $(p<0.05)$ and Heinz bodies $(\mathrm{p}<0.01)$. However, in the IVth attack patients, Hb levels correlated well with PCV and RBC ( $p<0.01)$, while it correlated inversely with parasite density $(\mathrm{p}<0.01), \mathrm{MCH}(\mathrm{p}<0.05), \mathrm{MCHC}(\mathrm{p}<0.05)$, osmotic fragility $(\mathrm{p}<0.01)$ and Heinz bodies $(p<0.001)$.

Fig. 2a, 2b, 2c, 2d. Effect of parasitemia on $\mathrm{MCH}, \mathrm{MCHC}, \mathrm{MCV}$ and Heinz bodies.

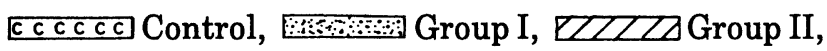
Group III, पाIDGroup III

a: Significantly different when compared to control.

b: Significantly different when compared to Group I.

c: Significantly different when compared to Group II.

d: Significantly different when compared to Group III.

Symbols represent statistical significance: *: $\mathrm{p}<0.05, @$ @ $\mathrm{p}<0.01$, \#: $\mathrm{p}<0.001$. 

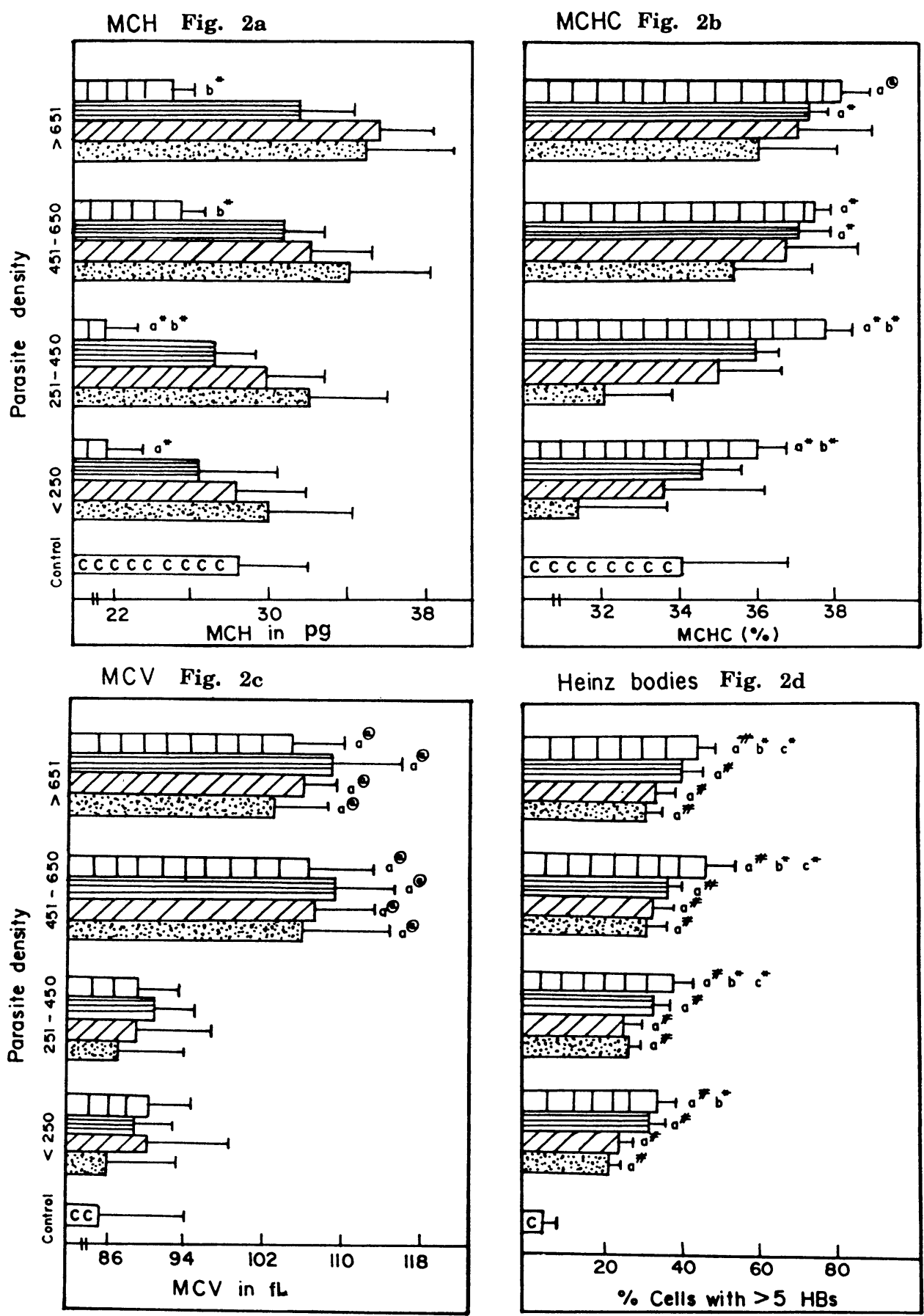

Heinz bodies Fig. 2d

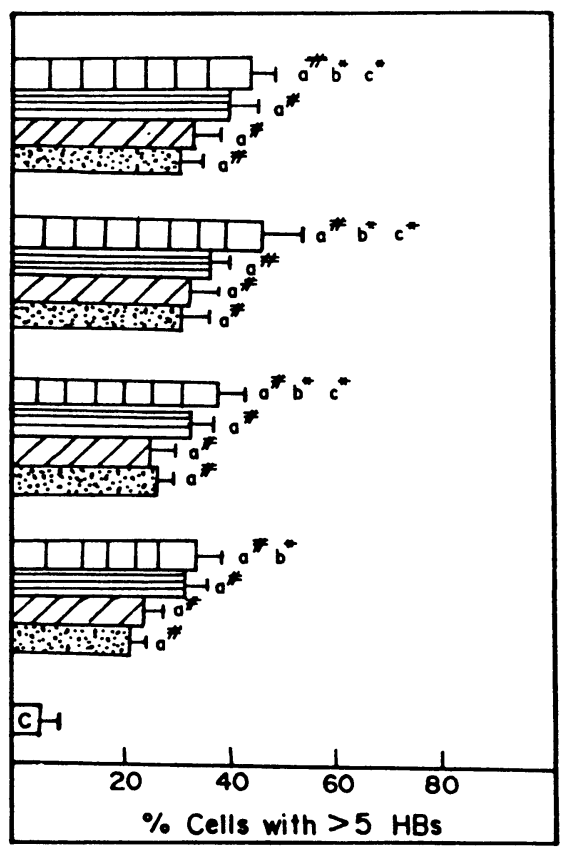


Table III. Correlations between hemoglobin and hematological indices in control and recurrent $P$. vivax malaria patients

\begin{tabular}{lccccc}
\hline Particulars & Control & Group I & Group II & Group III & Group IV \\
\hline $\begin{array}{l}\text { Parasite } \\
\text { density }\end{array}$ & - & -0.045 & -0.065 & $-0.312^{*}$ & $-0.405^{* *}$ \\
PCV & +0.094 & +0.173 & $+0.289^{*}$ & $+0.342^{*}$ & $+0.370^{* *}$ \\
RBC & +0.074 & $+0.294^{*}$ & $+0.339^{*}$ & $+0.369^{*}$ & $+0.422^{* *}$ \\
MCV & -0.053 & -0.402 & -0.423 & $-0.439^{*}$ & -0.430 \\
MCH & -0.042 & -0.152 & -0.221 & -0.362 & $-0.436^{*}$ \\
MCHC & -0.080 & -0.130 & -0.200 & -0.329 & $-0.459^{*}$ \\
$\begin{array}{l}\text { Osmotic } \\
\text { fragility }\end{array}$ & -0.065 & -0.309 & $-0.329^{*}$ & $-0.353^{*}$ & $-0.436^{* *}$ \\
Heinz bodies & -0.049 & $-0.349^{*}$ & $-0.358^{*}$ & $-0.442^{* *}$ & $-0.555^{* * *}$ \\
\hline
\end{tabular}

* - Statistical significance is shown at the level of $p<0.05$ ** - Statistical significance is shown at the level of $p<0.01$ *** - Statistical significance is shown at the level of $p<0.001$

\section{DISCUSSION}

In the present study, the degree of parasitemia has correlated with malarial incidence. This indicates that the parasitic load is comparatively less during the primary attack and progressed during further attacks. A similar observation has been reported in children from a malaria endemic area (12). This is attributed to the phenonmenon of malarial tolerance. It has been suggested that acquired malarial tolerance in humans operates through macrophages (13) and can provide a mechanism to limit the harm done by the parasite during the years it takes for acquired immunity to develop (14). Hence, the threshold of parasite density required to produce clinical symptoms during subsequent infections is elevated. This may be the possible reason for the direct correlation between parasitemia and number of malarial attacks. 
One of the salient features observed during malarial infection is hemolytic anemia. Woodruff et al. (15) have ascribed the cause of anemia in malaria to three factors: the destruction of erythrocytes by the parasites, the depression of erythropoiesis and probably the most important, hemolysis brought about by a complement-mediated immune process. During the development of the parasite, $\mathrm{Hb}$ is progressively digested and a concurrent release of high levels of ironcontaining breakdown takes place within the RBC (16). Indications for the progressive increase in redox-active iron causing oxidant stress has been observed during the growth of $P$. falciparum (17). Weiss (18) has stated that oxygen radicals traverse the RBC membrane by the anion channel and attack the intercellular $\mathrm{Hb}$. $\mathrm{Hb}$ metabolism is altered when the red cells are exposed to stress mediators with high redox potential. During malarial infection, which acts as a stressor for the red cell metabolism, there is raised levels of methemoglobin as evidenced in $P$. vivax patients (19).

Depression of erythropoiesis is another causative factor for decreased red cell production and subsequent anemia during malaria (20). Iron deficiency may also be implicated as the sole cause of dyserythropoiesis in patients with malarial anemia (21). The erythrocytes are destroyed by the parasite and the parasitized red cells are taken up by the phagocytes, which also engulf red cells not containing parasites. The erythropoietic centers are affected and bone marrow erythropoiesis is inhibited.

In the present study, both $\mathrm{MCHC}$ and $\mathrm{MCH}$ levels are decreased with increased MCV with repeated malaria infections suggesting macrocytic hypochromic anemia. On the other hand, increased parasitemia increases MCHC as well as MCV suggesting macrocytic hyperchromic anemia. Increased MCHC has been observed in experimental malaria due to spherocytosis which is frequently seen in immunohemolytic anemia (22). In addition, the biochemical abnormalities of the RBC and its membrane due to intracellular parasitism can increase hemolysis.

In the Heinz body inclusion test, no Heinz bodies are detected in the normal or malarial RBC. However, with the addition of acetylphenyl hydrazine, increased number of RBCs with more than five Heinz bodies are detected in the repeatedly infected malarial patients in comparison to the healthy controls.

Oxidative damage to $\mathrm{Hb}$ leads to formation of methemoglobin and reversible and irreversible hemichromes which precipitate and form Heinz bodies (23). It has been suggested that the physical presence of these Heinz bodies in the erythrocytes reduces the deformability of the cell, and binding of these inclusion 
bodies to the erythrocyte membrane results in osmotic damage with consequent lysis (24). The deleterious effect of irreversible hemichrome on red cell membrane is mediated by the release of free hemin, which may play a role in the destruction of erythrocytes in the so-called Heinz body containing hemolytic anemia with unstable $\mathrm{Hb}$.

Susceptibility to hypoosmotic shock is more pronounced in the erythrocytes of the repeatedly infected malaria patients. The osmotic fragility of both parasitised and nonparasitised RBCs are shown to increase significantly during malarial infections (25). Further, lowered filterability (26) and increased fragility (27) have been reported in the erythrocytes of experimental animals afflicted by malaria. The increase in osmotic fragility during malarial infection may be attributed to factors such as altered membrane permeability and increase in the volume to surface area ratio of erythrocytes (28). Studies with $P$. berghei-infected mice and $P$. falciparum-infected patients have shown increased lysis in hypotonic saline and lowered deformability index than normal cells (29).

The shift of the osmotic fragility curve to the right is indicative of the fragility of the erythrocytes and serves as an index to gauge the severity of the hemolytic process. Jacob and Lux (39) have proposed that peroxidative damage subsequently leads to the formation of a hole in the erythrocyte membrane. The increased erythrocyte fragility observed in our study can duly be ascribed to the leakiness of the membrane casued by peroxidation (3). Conceivably, the degree of leakiness is proportionate to the number of attacks. According to Seed and Kreier (31), the imbalance of the ionic concentrations $\left(\mathrm{Na}^{+}, \mathrm{K}+\right.$ and $\left.\mathrm{Ca}^{2+}\right)$ during malarial infection is responsible for the increase in volume and osmotic fragility of erythrocytes.

The above observations point to the fact that repeated malarial infections affect the blood profiles to a very great extent. It can possibly be suggested that recurrent Plasmodium vivax patients are prone to develop anemia in due course of the infection. Our future studies are aimed at overcoming the erythrocyte metabolic derangements in recurrent Plasmodium vivax patients, by using new antimalarial regimens.

\section{ACKNOWLEDGMENTS}

G. Baskaran wishes to thank Birla Smarak Kosh, Bombay, India, for their financial assistance. 


\section{REFERENCES}

1. Clark, I. A., Hunt, N. H., Cowden, W. B., Maxwell, L. E. and Mackie, E. J. (1984): Radical - mediated damage to parasites and erythorocytes in Plasmodium vinckei - infected mice after infusion of t-butyl - hydroperoxide. Clin. Exp. Immunol., 56, 524-530.

2. Goldberg, B., Stern, A. and Peisach, J. (1976): The mechanism of superoxide generation by the interaction of phenylhydrazine with haemoglobin. J. Biol. Chem., 251, 3045-3051.

3. Suresh, T. M. and Selvam, R. (1991): Effect of radical treatment on erythrocytes lipid peroxidation in Plasmodium vivax - infected malaria patients. Biochem. Mol. Biol. Int., 25, 211-220.

4. Drabkin, D. L. R. and Austin, J. H. (1932): Spectrophotometric studies, spectrophotometric constants for common haemoglobin derivatives in human, dog and rabbit blood. J. Biol. Chem., 98, 719-733.

5. Wintrobe, M. M. (1933): Macroscopic examination of the blood. Am. J. Med. Sci., 135, 58.

6. John, M. B. (1972): Appendix-Methods. p.1198-1204. In Laboratory Medicine Haematology, 4th ed., C. V. Mosby Co., St. Louis.

7. Wolf, P. L., Ferguson, P., Mills, I. T., Von Der Muehll, E. and Thompson, M. (1973): Erythrocytes: Absolute indices. p.250-252. In P. L. Wolf (ed.), Practical Clinical haematology Interpretation and Techniques. John Wiely and Sons, Inc., New York.

8. Miale, J. B. (1972): Appendix-Methods. p.1200-1201. In Laboratory Medicine Haemotology, 4th ed., C. V. Mosby Co., St. Louis.

9. Dacie, J. V. (1960): The congenital anemias. p.35-42. Hemolytic anemias, Part I. 2nd ed., Grune and Stratton, Inc., New York, NY.

10. Bauer, J. D., Ackermann, P. G. and Toro, G. (1974): Methods in haematology. p.85-144. In Clinical Laboratory, 8th ed., C. V. Mosby Co., St. Louis, MO.

11. Seshadri, C., Ramakrishna Shetty, B., Gowri, N., Sitaraman, R., Revathi, R., Venkataraghavan, S. and Chari, M. V. (1983): Biochemical changes at different levels of parasitaemia in Plasmodium vivax malaria. Ind. J. Med. Res., 77, 437-442.

12. McGregor, I. A., Gilles, H. M., Walters, J. H., Davies, A. H. and Pearson, F. A. (1956): Effects of heavy and repeated malarial infections on Gambian infants and children; effects of erythrocytic parasitization. Br. Med. J., 2, 686692.

13. Clark, I. A. (1987): Monokines and lymphokines in malarial pathology. Ann. Trop. Med. Parasitol., 81, 577-585.

14. Clark, I. A., Cowden, W. B. and Hunt, N. H. (1989): Malaria. p.702-722. In D. S. Nelson, (ed.), Natural Immunity. Academic Press, London.

15. Phillips, R. E., Looareesuwan, S., Warrell, D. A., Lee, S. H., Karbwang, J., Warrall, M. J., White, N. J., Swadichai, C. and Weatherall, D. J. (1986): 
The importance of anaemia in cerebral and uncomplicated falciparum malaria: Role of complications, dys erythropoiesis and iron sequestration. Q. J. M., 58, 305-323.

16. Woodruff, A. W., Ansdell, V. E. and Pettit, L. E. (1979): Cause of anaemia in malaria. Lancet, 1, 1055-1057.

17. Tai, G. and Ginsburg, H. (1993): Hemoglobin denaturation and iron release in acidified red cell lysate: A possible source of iron for intraerythrocytic malaria parasites. Exp. Parasitol., 77, 261-272.

18. Golenser, J., Marva, E., Hamic, M., Hempelmann, E., Cohen, A., Har-El, R. and Chevion, M. (1991): Free radicals and malaria. South Afr. J. Sci., 87, 584-587.

19. Weiss, S. J. (1982): Neutrophil-mediated methemoglobin formation in the erythrocyte. J. Biol. Chem., 257, 2947-2953.

20. Yuthavong, Y. (1985): Alterations of the erythrocyte membrane in malaria infection. J. Sci. Soc. Thai., 11, 53-65.

21. Yap, G. S. and Mary, M. S. (1994): Inhibition of in vitro erythropoiesis by soluble mediators in Plasmodium chabaudi AS malaria: Lack of a major role for interleukin 1, tumour necrosis factor alpha, and gamma interferon. Infect. Immun., 62, 357-362.

22. George, J. N., Wicker, D. J., Fogel, B. J., Shields, C. E. and Conrad, M. E. (1967): Erythrocytic abnormalities in experimental malaria. Military Med., 131, 1086-1090.

23. Peisach, J., Blumberg, W. E. and Rachemilevitz, E. A. (1975): The demonstration of ferrihemochrome intermediates in Heinz body formation following reduction of oxyhaemoglobin A by acetylphenylhydrazine. Biochem. Biophys. Acta, 393, 404-418.

24. Winterbourn, C. C. and Carrwell, R. W. (1974): Studies of hemoglobin denaturation and Heinz body formation in unstable hemoglobins. J. Clin. Invest., 54, 678-679.

25. Fogel, B., Shields, C. and Von Doenhoff, A. (1966): The osmotic fragility of erythrocytes in experimental malaria. Am. J. Trop. Med. Hyg., 15, 269-273.

26. Pattanakitsakul, S. and Yuthavong, Y. (1982): Heterogeneity infilterability of erythrocytes from malaria (Plasmodium berghei) - infected blood. Experientia, 38, 626-628.

27. Seed, T. M., Brindley, D., Aikawa, M. and Rabbege, I. R. (1976): Plasmodium berghei: Osmotic fragility of malaria parasites and mouse host erythrocytes. Exp. Parasitol., 40, 380-390.

28. Schrier, S. L., Basant, B. I., Benesh, K., Seegar, M. and Junge, I. (1974): Erythrocyte membrane vacuole formation in hereditary spherocytosis. Br. J. Haematol., 26, 59.

29. Dluzewski, A. R., Rangachari, K., Wilson, R. J. M. and Gratzer, W. B. (1985): Relatioin of red cell membrane properties to invasion by Plasmodium falciparum. Parasitology, 91, 273-280. 
30. Jacob, H. S. and Lux, S. E. (1968): Degradation of membrane phospholipids and thiols in peroxide hemolysis: Studies in vitamin E deficiency. Blood, 32, 549-568.

31. Seed, T. M. and Kreier, J. P. (1972): Plasmodium gallinaceum: Erythrocyte membrane alterations and associated plasma changes induced by experimental infections. Proc. Helminthol. Soc. Wash., 39, 387-411. 